\section{Big alcohol catches up with all adolescents}

Flegel has written an interesting and compelling editorial. ${ }^{1}$ Ensuring young women have knowledge about the dangers of alcohol and the skills to mitigate these dangers are reasonable goals. However, Flegel states "Female-specific risks are already well known and include violence, unwanted sex and pregnancy, and inadvertent alcohol consumption during early pregnancy." Since when did violence, unwanted sex and pregnancy become female-specific risks? Becoming critical consumers of advertising, having a clear understanding of the risks to judgment and behaviour associated with alcohol consumption, and ensuring the ability to create a solid foundation for healthy and safe sexual relationships are important for both young women and young men.

\section{Melissa Brouwers PhD}

Associate professor, McMaster University, Hamilton, Ont.

\section{Reference}

1. Flegel K. Big alcohol catches up with adolescent girls. CMAJ 2013;185:859.

CMAJ 2013. DOI:10.1503/cmaj.113-2131

\section{Concerns with fibromyalgia guidelines}

As a clinician with a practice dedicated to patients with fibromyalgia and related disorders, it is heartening to read about the advances in the research and clinical care of fibromyalgia over the past 2 decades and to see that information published in a widely read journal such as CMAJ. ${ }^{1}$ I support many of the recommendations in the 2012 Canadian guidelines, ${ }^{2}$ including the need to increase the capacity of primary care physicians to make an accurate diagnosis and initiate management of fibromyalgia, referring patients to specialists only if further expertise is required. I also agree that patients should be encouraged to take an active role in their own management and to engage in multimodal treatment. ${ }^{3}$ I would like to highlight 3 concerns with the new Canadian guidelines ${ }^{2}$ and their summary in $C M A J .{ }^{1}$

First, the article ${ }^{1}$ does not clearly state that although fibromyalgia and major depression can be comorbid, they are not the same condition and they do not respond to the same treatments. The authors show that selective serotonin reuptake inhibitors are ineffective in fibromyalgia. In contrast, these drugs are among the drugs of choice for depression. ${ }^{4}$ The effects of duloxetine on fibromyalgia appears to be independent of depression status or antidepressant effects, ${ }^{5}$ suggesting that pain and mood pathways are different.

Second, despite citing research that shows that the evidence for exercise effects in fibromyalgia is neither convincing nor sustained, the authors recommend exercise for all patients. Exercise is an important component of treatment, but it is not possible without exacerbating pain and other symptoms for some severely affected individuals. Many patients have told me that their family doctor has told them to "exercise" but has not provided any guidance as to how to do it safely or effectively. I suggest starting at a modest intensity (e.g., $65 \%$ of age-adjusted maximum heart rate) and increasing slowly by $10 \%$ per month, as tolerated and sustained. ${ }^{6}$ In my experience, forced graded exercise, irrespective of symptom exacerbation, is not effective.

Third, one of the most perplexing issues in fibromyalgia is how to reconcile the healthy appearance of many patients with their subjective disability levels. However, the absence of objective markers for a condition is not the fault of the patient and they should not be disbelieved or penalized because of it. Physicians have the responsibility to support the needs (disability and otherwise) of patients with poorly understood conditions with the same professional ethic they have for patients with better understood conditions.

\section{Eleanor Stein MD}

Psychiatrist, University of Calgary, Calgary, Alta.

\section{References}

1. Fitzcharles M-A, Ste-Marie PA, Pereira JX Fibromyalgia: evolving concepts over the past 2 decades. CMAJ 2013;185:E645-51.

2. Fizcharles M, Ste-Marie PA, Goldenberg D, et al. Canadian Guidelines for the diagnosis and management of fibromyalgia syndrome. Oshawa (ON): Canadian Pain Society; 2012:1-44.

3. Let your light shine through: strategies for living with myalgic encephalomyelitis/chronic fatigue syndrome, fibromyalgia and multiple chemical sensitivity. Calgary (AB): Eleanor Stein; 2012.

4. Cipriani A, Furukawa TA, Salanti G, et al. Comparative efficacy and acceptability of 12 new-generation antidepressants: a multiple-treatments metaanalysis. Lancet 2009;373:746-58

5. Arnold LM, Lu Y, Crofford LJ, et al. A doubleblind, multicenter trial comparing duloxetine with placebo in the treatment of fibromyalgia patients with or without major depressive disorder. Arthritis Rheum 2004;50:2974-84.

6. Jones KD, Clark SR. Individualizing the exercise prescription for persons with fibromyalgia. Rheum Dis Clin North Am 2002 May;28:419-36, x-xi

CMAJ 2013. DOI:10.1503/cmaj.113-2132

\section{The authors respond}

We thank Stein ${ }^{1}$ for her interest in our $C M A J$ article, ${ }^{2}$ which reflects concepts addressed by the 2012 Canadian fibromyalgia guidelines.

We agree with Stein that major depression and fibromyalgia are distinct disorders, however there is considerable overlap. Evaluating patients with fibromyalgia for mood disorders, including depression, is imperative and not doing so could compromise optimal patient care. The effects of an antidepressant on pain have been shown to be independent of effects on mood for a single agent only. ${ }^{3}$ Nevertheless, the choice of an agent that may address more than 1 symptom should be considered beneficial. In the guidelines, ${ }^{3}$ recommendations pertaining to antidepressant use in fibromyalgia state that "all categories of antidepressant medications including TCAs, SSRIs and SNRIs may be used for treatment of pain and other symptoms in patients with fibromyalgia," and that physicians should explain the pain-modulating effects of antidepressants to patients. ${ }^{3}$

With regard to exercise, we have difficulty accepting a report of disablement to be so severe as to preclude any form of health-related physical activity. Regular physical activity is recommended for all persons, irrespective of health status. ${ }^{3}$ The guidelines focus on the importance of a patient-tailored 